Macedonian Pharmaceutical Bulletin, 66 (Suppl 1) 187 - 188 (2020)

Online ISSN 1857 - 8969

UDC: 613.292

DOI: 10.33320/maced.pharm.bull.2020.66.03.093

Short communication

\title{
Safety aspects of products, food supplements, intended for weight loss treatment
}

\author{
Blagica Samarova Stoev*, Dafinka Damcevska, Silvija Saveska, Tatjana Bogovska, Nada \\ Stojanoska, Mena I. Zdravkovska, Hristina Babunovska
}

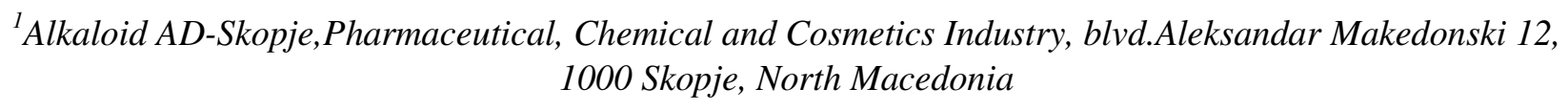

\section{Introduction}

Nutrition supplements are food products that supplement the normal diet and are concentrated sources of nutrients or other substances with a nutritional or physiological effect, alone or in combination, released in dosage form as capsules, lozenges, tablets, sachets with powder substance, liquid vials and other similar forms designed to be used in small quantifiable quantities. This product group includes vitamins, minerals, amino acids, enzymes, pre and pro biotics, essential fatty acids, herbs and extracts, mushrooms, algae, various bioactive substances.

The pharmacodynamic effects of the supplements are due to the presence of certain active ingredients in them. Efficacy is based on centuries of experience since there are few well-controlled, randomized, clinical trials.

Overview of the regulatory framework on the safety aspects of products and food supplements intended for weight loss treatment

The increasing number, as well as the consumption of nutrients and other ingredients that can be presented as dietary supplements and the different national rules for their marketing have led to the adoption of Directive 2002/46 / EC within the EU. The Directive represents:

- Harmonized regulatory framework for national law for EU Member States;

- Contains a harmonized list of vitamins and minerals that can be added for nutritional purposes in food supplements; Annex I - types of vitamins and minerals; Annex II - Form and purity of substances;

- Harmonized rules for labeling supplements whether it has a health or nutritional label.

Directive 96/8 / EC regulates food intended for energy-restrictive diets for weight loss. According to this directive, our "Rulebook on food safety intended for use in restricted-value diets to reduce weight" has been developed. Rulebook foods intended to be used in diets with a limited energy value for weight loss purposes are foods with a specific composition which, if used in accordance with the instructions given by the manufacturer in whole or in part, replaces overall daily meals.

Foods with limited energy value are divided into two categories:

1. Food that is presented as a complete replacement of daily meals;

2. Food that is presented as a substitute for one or more daily meals.

\footnotetext{
*bsamarova@alkaloid.com.mk
} 
EFSA represents EU pillar on risk assessment and food safety on the territory of European Union.

The DSHEA is a FDA-law on US territory that regulates dietary supplements since 1994. This law requires any supplement to be marked as such; provide reasonable evidence (expectations) of safety, but does not require the manufacturer to have FDAapproved product safety prior to being placed on the market. Once the dietary supplement is released to the market, the FDA has the responsibility to monitor product safety through mandatory reporting of adverse events by manufacturers, consumers and health professionals.

\section{Challenges and perspectives}

Weight loss supplements contain many ingredients such as minerals, vitamins, amino acids, enzymes, fiber, herbs in various amounts and in many combinations in the form of capsules, tablets, liquids or powders. The main ingredients of weight loss supplements are: African Mango, Beta Glucani, Bitter orange, Caffein, Calcium, Capsaicin, Carnitin, Chitosan, Chromium, Coleus forskohlii, Conjugated linoleic acid, Fucoxanthin, Gasinia cambogia, Glucomanan, Green coffee bean extract, Green tea, Guar gum, Hoodia, Probiotics, Pyruvate, Rasberry ketone, Vitamin D, White kidney bean, Yohimbe.

Most products contain more than one ingredient, and the ingredients can function differently if combined. Therefore, it is actually complicated to discover the safety of these preparations during use for their intended purpose, weight loss. There can be safety evidence for only one ingredient in the finished product, and no evidence of the efficacy and safety of others is available. In addition, the doses and the amount of active ingredients vary greatly among weight loss supplements, and the composition of the product is not always fully described in published clinical trials.
Manufacturers of weight loss supplements rarely conduct clinical studies in humans to find out the efficacy and safety of the product. Even when clinical trials are done, they usually involve a small number of subjects using the product for a short period of only a few weeks or months. Studies may also use different and sometimes inappropriate techniques to evaluate the efficacy and safety of a preparation.

\section{Conclusion}

Concerning the safety of supplements, there are limited data, without preclinical studies, an insufficiently known safety profile, the occurrence of drug interactions that emphasize or diminish its effect, the effect of absorption, metabolism or excretion of the drug or the occurrence of adverse drug reactions. allergic reactions, GIT disorders, liver, kidney, CNS disorders, sedation, depression, etc.

Natural, doesn't always mean safe and secure.

\section{References}

https://www.nowfoods.com/now/nowledge/fda-anddietary-supplements-how-supplements-areregulated.

http://fva.gov.mk/images/stories/hrana/pravilnik_nut ritivna 3.pdf.

https://ec.europa.eu/food/safety/labelling nutrition/s pecial_groups_food/weight_reduction_en. https://ods.od.nih.gov/HealthInformation/DS_What YouNeedToKnow.aspx. https://ods.od.nih.gov/factsheets/WeightLossHealthProfessional/. https://eur-lex.europa.eu/legal-content/. 